\title{
Power Posture Effects on Approach and Avoidance Decisions in Response to Social Threat
}

Short title: Posture effects on social action decisions

Hannah Metzler ${ }^{\star 1,2,3}$, Emma Vilarem ${ }^{1}$, Adrian Petschen ${ }^{1,4}$, Julie Grèzes ${ }^{\star 1}$

1. Cognitive and Computational Neuroscience Laboratory, INSERM unit 960, Ecole Normale Supérieure, PSL University, Paris, France.

2. Sorbonne Universités, UPMC University Paris 06, Paris, France

3. Institute for Globally Distributed Open Research and Education

4. Institut de la communication et de la cognition, Université de Neuchâtel, Neuchâtel, Switzerland.

Current addlaress: Hannah Metzler is now at Complexity Science Hub Vienna, Josefstaedter Strasse 391080 Vienna, Austria, metzler@csh.ac.at (instead of affiliation 1 and 2 listed above).

* Corresponding authors

E-mail: metzler@csh.ac.at (HM), julie.grezes@ens.fr (JG) 


\begin{abstract}
Individuals' opportunities for action in threatening social contexts largely depend on their social power. While powerful individuals can afford to confront aggressors and dangers, powerless individuals need others' support and better avoid direct challenges. Here, we investigated if adopting expansive or contracted postures, which function as social signals of power, impacts individuals' approach and avoidance decisions in response to social threat signals using a within-subject design. Overall, participants more often chose to avoid rather than to approach angry individuals, but showed no clear approach or avoidance preference for fearful individuals. Crucially, contracted postures considerably increased the tendency to avoid angry individuals, whereas expansive postures induced no substantial changes. This suggests that adopting power-related postures may impact action decisions in response to social threat signals. The present results emphasize the social function of power postures, but should be replicated before drawing strong conclusions.
\end{abstract}

Keywords: power posture, approach, avoidance, emotion, threat, face perception 


\section{Introduction}

In everyday life, we continuously gather information from our physical and social environment to flexibly adapt our behaviour. Among social signals, which we integrate in daily action decisions, emotional expressions of others are of particular relevance. Facial displays of emotion, notably threat displays, prompt motivational orientations that prepare the organism for appropriate responses. The perceived available action opportunities in response to social threat signals are, however, constrained by the individual's action capabilities (Frey \& Grafton, 2014), which are notably determined by their power and dominance status. Powerful individuals can afford to approach aggressive others and confront them, whereas powerless individuals may be better off avoiding conflicts and seeking social support as a means of protection. This study investigated whether adopting power-related postures impacts individuals' approach and avoidance decisions in response to social threat signals.

In many social species, such as chimpanzees, rodents, birds, wolfs, dogs, and crickets, power-related behaviours are closely linked to bodily expansiveness (de Waal, 2007; Grant \& Mackintosh, 1963; Hagelin, 2002; Schenkel, 1967; Stevenson et al., 2000). Large body size implies physical strength and often signals threat, whereas small bodies signal submission and vulnerability (Mehrabian, 1981; Schuett, 1997; Sell et al., 2009; Tokarz, 1985). Humans use analogous non-verbal displays to express social power and dominance, for example following competitive interactions (Hwang \& Matsumoto, 2014). Expansive body postures signal high dominance, power (Hall et al., 2005) and success (Tracy \& Matsumoto, 2008; Weisfeld \& Beresford, 1982), whereas contracted postures convey low power and social status, as well as defeat.

Building on theories of embodiment, several studies have examined whether body expansiveness, in addition to signalling social power, could also influence the individuals' own feelings, moods and behaviour (Duclos et al., 1989; Riskind, 1984; Riskind \& Gotay, 1982; Stepper \& Strack, 1993). Although the replicability of most studies exploring the impact of adopting expansive and contracted postures was subject to an intense debate (Jonas et al., 2017; Smith \& Apicella, 2017), there seems to be evidence for a small effect of adopting expansive or contracted postures on explicit feelings of power (Gronau et al., 2017). Yet, feelings as well as many other investigated behaviours were assessed using explicit self-reports, which are susceptible to demand effects (Gronau et al., 2017; Jonas et al., 2017). Past research on other behaviours typically focused on non-social but power-related behaviour such as risky gambling, abstract and creative thinking (Andolfi et al., 2017; Carney et al., 2010; Cesario \& McDonald, 2013; Huang et al., 2011), or cognitively complex social behaviour, such as sales negotiation, cheating or planning to take revenge or to volunteer (Cesario \& Johnson, 2017; Strelan et al., 2014; Yap et al., 2013). Importantly, this selection of behaviours neglects the elementary and social signalling function of postural expansiveness apparent in many animal species. The only existing study on low-level social behaviour, namely visual dominance behaviour and speaking time, reported no 
significant effects, but was statistically underpowered $(n=22, d=0.26$ for visual dominance ratio; Jamnik \& Zvelc, 2017). Accordingly, investigations of effects of powerrelated postures on social perception and associated behaviours remain scarce.

Both current power status (Schultheiss \& Hale, 2007; Yap et al., 2013) and socially meaningful body actions (Niedenthal, 2007) have the potential to distort our perception of the social world. Consistent with this, adopting power-related postures also appears to impact social threat perception. A recent study shows that an observer's body posture may determine the salience of task-irrelevant emotional facial displays (Chadwick et al., 2019). Participants had to discriminate between in- and outdoor scenes, which were superimposed on faces expressing anger or fear, together with either direct or averted gaze. Participants who had adopted an expansive posture were most accurate in discriminating scenes when they were superimposed on angry expressions with direct gaze, which signal a dominance challenge towards the observer. In contrast, participants who had adopted a contracted posture performed best in the presence of fearful expressions with averted gaze, which signal danger in the environment. Although this indicates that observers' body posture could influence how they perceive social threat signals, it is currently unknown whether it also influences the observers' action decisions in response to such signals.

Here, we investigated whether adopting power-related postures would influence spontaneous action choices in the presence of task-irrelevant facial displays. We focused on threat-related facial expressions of anger and fear, given that power most crucially determines individuals' action opportunities in threatening contexts. Moreover, angry and fearful faces, although both being of negative valence, convey different social meanings (Sander et al., 2007; Springer et al., 2007) and elicit different reactions (Marsh et al., 2005; Vetter et al., 2019). We used a novel approach-avoidance task developed by Vilarem et al. (2019) that allows studying participants' spontaneous action decisions in a realistic social context. The stimuli depict a waiting room with four chairs, of which the outer two are empty. The two middle chairs are occupied by two taskirrelevant individuals, one displaying a neutral expression while the other displays either a neutral, fearful or angry expression. Participants freely chose where to sit by moving the mouse cursor to the chair of their choice, thereby either approaching or avoiding the emotional individual. Past studies with this task (Mennella et al., 2020; Vilarem et al., 2019) have found that participants choose to avoid angry individuals more often than fearful ones.

To assess postural feedback effects, we ran two consecutive sessions of this task: the first session, without postures, served as within-subject baseline, whereas participants adopted either an expansive or a contracted posture before each task block in the second session. We expected the adopted postures to impact action decisions in the presence of task-irrelevant threat displays according to the level of power they embody. Greater physical and social resources (Keltner et al., 2003) enable powerful individuals to better cope with social threat, such as an angry opponent. Moreover, power increases approach motivation in general (Guinote, 2017; Keltner et al., 2003) and decreases vigilance towards threat (Anderson \& Berdahl, 2002; Willis et al., 2011). 
Similarly, manipulations of power induce opposite approach and avoidance tendencies in response to sustained direct eye gaze, another social threat and dominance signal (Weick et al., 2017). Therefore, if expansive postures embody high power, they should decrease avoidance of angry individuals, in contrast to contracted postures, which should increase avoidance. Our predictions for fearful displays were more speculative, given that they simultaneously signal the presence of danger (Paulus \& Wentura, 2016) and enhance facial cues of vulnerability and affiliation (Hess et al., 2000; Knutson, 1996; Marsh et al., 2005; Sell et al., 2014). If participants are more sensitive to the threat aspect after a contracted than after an expansive posture, one should expect stronger avoidance for fearful displays. If, however, participants are more sensitive to the affiliative aspect of fearful displays, a different pattern should emerge. Specifically, lack of power increases affiliation motivation (Case et al., 2015) and subtle cues of low as compared to high social status increase pro-social behaviour (Guinote et al., 2015). Hence, contracted as compared to expansive postures may increase approach towards fearful individuals, who signal a need for help and represent potential allies in the defence against threat.

Previous studies with the same approach-avoidance task (Vilarem et al., 2019) found that both angry and fearful faces elicited similar and quicker reactions than neutral faces, hinting that the increased avoidance of angry faces cannot be explained by more efficient processing. Instead, as the results by Menella et al. (2020) further confirm, the emotional displays seem to modify the value of each action possibility and orient the decision process toward the better outcome (i.e. avoiding the angry individual). In parallel, we expected that postures would not influence the ability to detect anger or fear (as indicated by movement kinematics), but modify the value of the avoidance action.

\section{Methods}

Data, code and material to reproduce the below procedures and analyses are available at https://osf.io/q8s3w/.

\section{Participants and Power Analysis}

A total of 88 healthy, right-handed, fluently French speaking men between 17 and 35 years old were recruited via a mailing list and online student job platforms. All participants had normal or corrected vision, were not currently under medical treatment, and did not suffer from ocular pathologies or ocular fatigue in front of a screen. The experimental protocol was approved by INSERM and the local research ethics committee (Comité de protection des personnes Ile de France III - Project C07-28, N Eudract: 207-A01125-48), and was carried out in accordance with the Declaration of Helsinki. Participants provided informed written consent and were paid for their participation.

In the context of the debate around the replicability of previous power posing studies (this study began in 2016), a major priority was to achieve high power within our 
feasibility constraints. A re-analysis of two important power posing studies (Credé \& Phillips, 2017) as well as an exploratory analysis in a highly powered study (Bombari et al., 2017), hinted that the poses' effect on feelings may be stronger in men than in women. Similarly, in a study investigating the discrimination of angry and fearful facial expressions (Metzler, 2018), we observed a slightly stronger posture effect in men compared to women, although the respective interaction effect was not significant $(p=.07)$. We therefore decided to prioritize high power over gender balance in this first study on social action decisions, and included only male participants. We calculated the necessary sample size for detecting a significant posture by emotion interaction on the change in proportion of away choices from the first to the second session at an alpha of 0.05 and 0.80 power. We based the calculation on the smaller out of two previously observed effects of emotion in the same task, i.e. partial eta-squared of $\eta_{p}^{2}=0.30$ compared to $\eta_{p}^{2}=0.36$ (Mennella et al., 2020; Vilarem et al., 2019). Entering this effect size (as $\mathrm{f}=0.65$ ) in a one-way repeated measures ANOVA in $\mathrm{G}^{*}$ Power (Faul et al., 2007) yielded a minimal sample size of $n=22$ to detect the emotion effect, which corresponds to 22 per group to detect the interaction with posture if posture completely suppressed the effect in one group (Simonsohn, 2015). Yet, we expected the emotion effect to be larger in the contracted posture and smaller in the expansive posture, with the real extent of this change being unknown. Therefore, we doubled this number, aiming at 44 participants per posture group.

The final sample consisted of 79 participants (see section on data cleaning) with a mean age of $22.70 \pm 3.64$ years, of which 40 and 39 had been randomly assigned to the expansive and contracted condition, respectively. We conducted a sensitivity analysis to determine the minimal effect-size we could reliably detect with a power of $80 \%$ using the final sample size of $n=79$. This yielded a minimal effect size $\eta_{p}^{2}=0.10(f=0.33$ in $G$ Power), meaning we had at least $80 \%$ power for effects larger than this, and less than $80 \%$ for smaller effects.

\section{Stimuli and Task}

Stimuli (Vilarem et al., 2019) were presented using Psychophysics Toolbox 3 (Brainard, 1997; Kleiner et al., 2007) in MATLAB version 2014b (MathWorks, Inc., 2014). They depicted a waiting room with four chairs, with two individuals seated on the two chairs in the middle. One of the two depicted individuals always displayed a neutral facial expression while the other displayed, in one third of the trials, either a neutral, angry, or fearful expression. The stimuli included facial expressions from 10 different pairs of individuals from the Radboud Faces Database (Langner et al., 2010), which were matched for gender as well as for perceived threat and trustworthiness (Vilarem et al., 2019). Expressions of anger and fear varied along 4 levels of intensity (morphs between the neutral and the full emotional expression), which were equalized for perceived emotion intensity (El Zein et al., 2015). The identities within each pair of individuals as well as the side (left/ right) of the individual expressing an emotion were fully counterbalanced. This resulted in a total of 480 trials per session: 10 pairs $\times$ ( 2 
emotions $\times 4$ intensity levels +1 neutral expression $\times 4$ repetitions) $\times 2$ identities displaying the emotion $x 2$ sides of the emotional individual.

Participants' task was to choose on which of the two outer chairs they wanted to sit, by moving the mouse cursor from the middle of the screen to one of the two outer chairs. Throughout the trial, they had to keep their eyes on the fixation cross displayed between the two faces. They were told to choose the chair as if making the choice in a real situation, and that there was no good or bad choice, as long as they released the cursor within one of the chair areas. Participants were asked to maximize the number of valid trials, i.e. to land within one of the chair areas within 1400ms. Their accuracy score (percentage of valid trials) was displayed at the end of each block. The timing of a trial is shown in Figure 1.

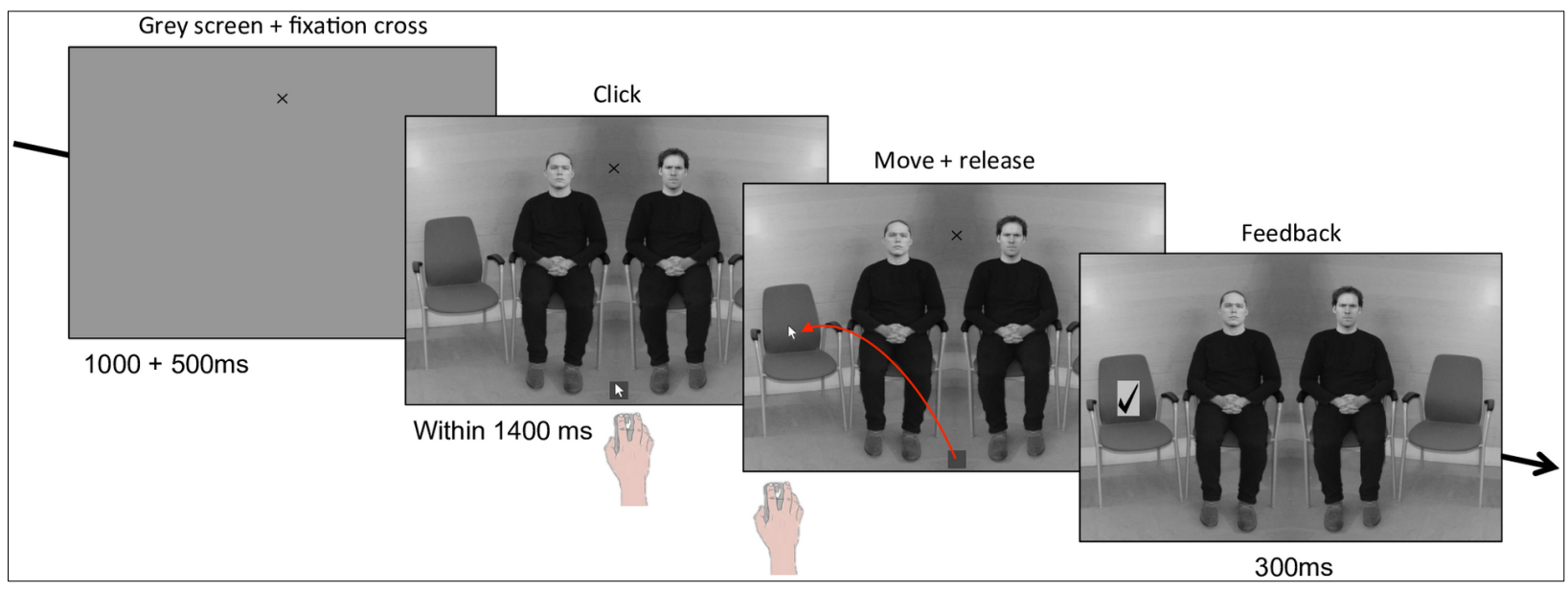

\section{Fig 1. Stimuli and task.}

Each trial started with a grey screen of $1000 \mathrm{~ms}$, onto which a fixation cross was then superimposed for $500 \mathrm{~ms}$, followed by the scene with the mouse cursor at the bottom centre. Throughout the trial, participants were to focus on the fixation cross displayed between the faces. Upon presentation of the waiting room, they had to click and then hold and move the mouse cursor from the grey square to the chair of their choice, and release it within the chair area. In valid trials, i.e. when releasing the cursor within the chair area within 1400ms after scene onset, a tick appeared at the release location for $300 \mathrm{~ms}$ before the next trial started. In case of releases outside of the chair area, the next trial started immediately, or after $1400 \mathrm{~ms}$ if no response occurred.

Participants were seated at a distance of $60 \mathrm{~cm}$ from the eyes to the screen so that the eccentricity to the central fixation cross was of 4.5 degrees for the centre of the faces, and of 8 degrees for the centre of the chairs. The experimenter emphasized the importance of fixating the fixation cross throughout the trial and never mentioned that the depicted individuals were going to express any emotions. Participants underwent a short training session with neutral facial expressions only, during which a blue rectangle indicated the area on top of each chair until they correctly landed on the chair in at least $60 \%$ of trials. In total, there were 10 blocks of 96 trials with a mean duration of $6.5 \pm$ 1.95 SD minutes. Participants reached mean accuracy scores of $91.3 \% \pm 5.37$ SD. 


\section{Procedure}

To check if relevant trait or state differences between the two posture groups did not contribute to any observed group difference, participants completed questionnaires before the testing day, and just after signing consent forms (see the SI for further details and results). Participants performed the first 5 task blocks without posture (session 1), and then stood in either an expansive or contracted posture (see Fig 2) for 2 minutes before each of the next 5 task blocks (session 2).

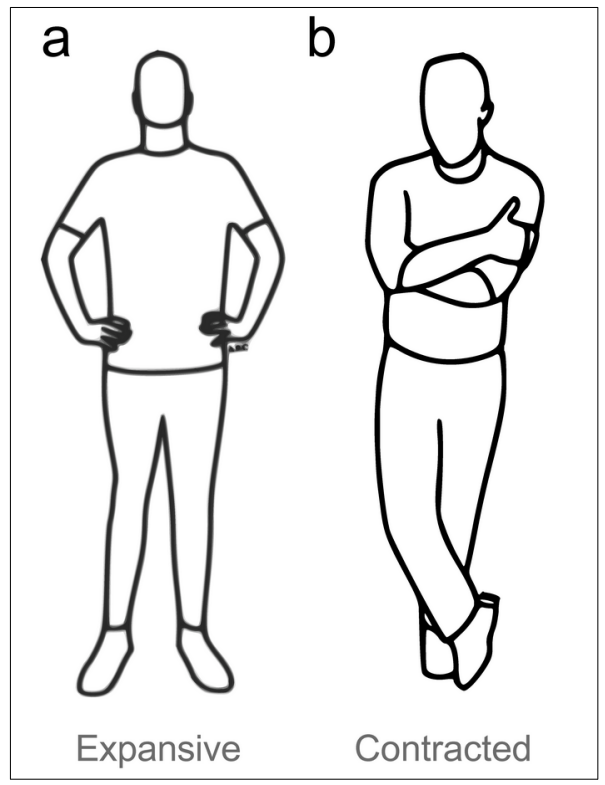

Fig 2. Postures adopted by each of the experimental groups.

Participants adopted no posture in session 1 (baseline). In session 2, they adopted one of the depicted postures for 2 minutes before each of 5 task blocks. (a) Expansive posture. (b) Contracted posture. Legend: Images created by Antoine BaloukaChadwick.

To justify this experimental design, the male experimenter told participants that they were participating in two separate studies, the first investigating spontaneous action choices and the second posture effects on heart rate. The cover story further mentioned several advantages of combining the two studies, such as breaks from screen-time in the second half of the experiment and short posturing intervals to avoid discomfort. After session 1, the experimenter attached electrodes to participants' hand-wrists and demonstratively turned on the heart rate acquisition system, although no data were recorded. To minimize possible experimenter biases, participants were randomly assigned to a posture condition just before posture instructions were provided (see SI for more details on instructions).

Careful debriefing at the end of the experiment suggested that only 12 participants had noticed an influence of facial expressions on their decisions, none could specifically identify anger and fear as the two displayed emotions, and only 2 participants suspected a link between the posture and the task. Excluding these 2 participants did not change the results. Such low familiarity with power posing may be due to the fact 
that all sessions were conducted between November 2016 and March 2017, when media coverage on power poses in France was low. For more details, see the SI.

\section{Data Analysis}

After exporting data from Matlab, all analyses were conducted in $R$ version 3.4 .4 ( $R$ Core Team, 2018) using the packages dplyr, tidyr, ez, ggplot2 and psych (Lawrence, 2016; Revelle, 2017; Wickham, 2009; Wickham et al., 2017; Wickham \& Henry, 2018), in addition to those specifically mentioned below. We recorded which chair was chosen (coded as moving away from or toward the emotional individual), and the movement kinematics initiation time (time from scene onset to mouse click) and movement duration (time from mouse click to release within the chair area).

Data cleaning. Only valid trials were included in the analysis (landing on top of a chair area and under $1400 \mathrm{~ms}$ ). Additionally, we removed two types of trials which reflected lack of compliance with task instructions: trials in which participants anticipated the scene onset due to the fixed duration of the inter-trial interval and clicked too early (trials with initiation times below the 5th percentile, i.e. below 95ms) and trials in which participants clicked after the beginning of the movement (trials for movement durations below the 5th percentile, i.e. shorter than $184 \mathrm{~ms}$ ). To ensure a sufficient number of trials per participant, we required at least $50 \%$ of valid trials per session above the respective 5th percentile threshold for both initiation time and movement duration, which entailed the exclusion of 6 participants. Excluding these participants, of which 3 were in the expansive and contracted condition, respectively, did not affect the significance of effects in the model of choice, initiation and movement time. Due to a technical error, data from 2 further participants was lost. Another participant was excluded because he did not achieve 60\% accuracy after repeating the training three times (see task description). Mean trial number per emotion condition (anger, fear, neutral) in each session for all remaining 79 participants was $132.6 \pm 17.22$ with a range of 65-158 trials. Finally, initiation time and movement duration were log-transformed to correct for the obvious right skew typically observed in reaction time distributions. All 95\% confidence intervals in figures and tables are within-subject intervals calculated separately for each group according to the Cousineau-Morey method (Baguley, 2012) in order to highlight pre-post posture changes.

Proportion of choice. Analysis of choice (away vs. toward), and movement kinematics was performed using linear mixed-effects models (LMEM) as implemented in the Ime4 package (Baguley, 2012) to account for repeated-measures and the unbalanced design (unequal number of participants per posture and valid trials per condition), and to consider random variation between participants as well as stimuli. For choice, we analysed effect of emotion (anger vs. fear, within-subject), and session (session 1 baseline vs. session 2 with posture, within-subject) and posture (expansive vs. contracted, between-subject). We chose not to examine the effect of intensity because we had no particular hypothesis regarding intensity and posture, and models without intensity already involved interactions of three and four predictors for choice and kinematics, respectively. 
We used deviation coding $(-0.5,0.5)$ for emotion, with fear as the baseline category. In contrast, session and posture were treatment coded $(0,1)$ with session 1 and expansive as default baselines. With this combination of contrast coding, the model intercept corresponds to the mean of the dependent variable across emotion in the baseline category, i.e. in the expansive group in session 1. The estimate for emotion represents a main effect, whereas estimates for session and posture correspond to simple effects (i.e., they reflect the change from session 1 to 2, and the difference between posture groups). To obtain effect estimates for main-effects and two-fold interactions in the contracted group and session 2, we re-levelled posture and session and ran each model once with each posture and session as the baseline category.

We applied a generalized LMEM with binomial family and logit function on only angry and fearful trials (choice away or toward the emotional actor is not meaningful in neutral trials), with main effects and all interactions of emotion, session and posture as fixed effects, and a random intercept for participants and stimuli (see SI for more details on the random effects structure). To interpret our main result with regard to the power and sensitivity analyses, we further report an ANOVA of proportion of choice with session, emotion, and posture in the SI. Details on the analysis and the results regarding movement kinematics are reported in the SI.

\section{Results}

\section{Proportion of Choice}

A likelihood ratio test comparing the generalized LMEM choice $\sim(1 \mid$ subject $)+(1 \mid$ stimulus pair) + emotion $x$ session $x$ posture to the null model with only random intercepts, indicated a good fit to the data $\left(X^{2}(7)=35.789, p<0.001\right.$, deviance $(-2 L L)$ reduced from 57854 to 57818). Fig 3a illustrates that there was a higher probability of avoiding anger as compared to fear in both sessions and groups. However, the predicted three-fold interaction indicated that this effect of emotion on action choices (more avoidance for anger than fear) changed between sessions as a function of adopted posture $(\mathrm{OR}=1.19,95 \% \mathrm{Cl}=1.02-1.38, \mathrm{z}=2.18, \mathrm{p}=0.029)$. Specifically, the difference in avoidance of anger and fear was larger in the second than the first session in the contracted as compared to the expansive posture. 

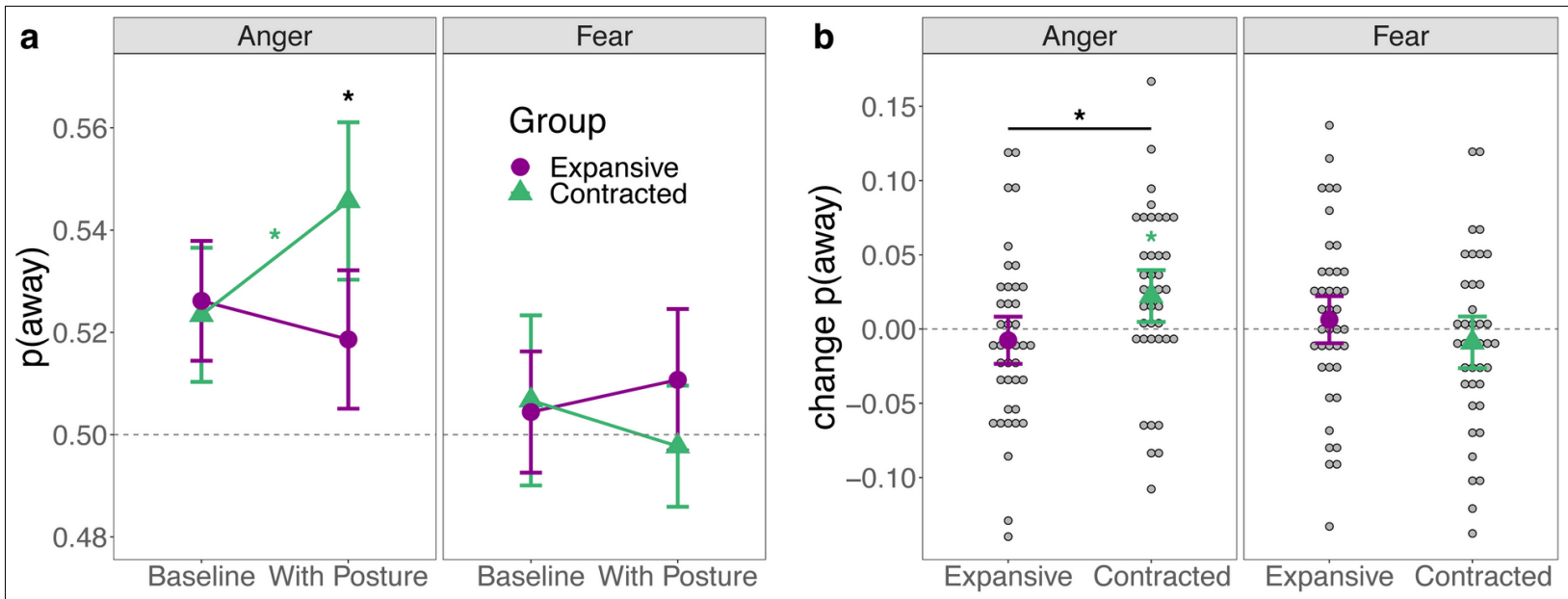

Fig 3. Means and within-subject confidence intervals for proportion of choice.

$\mathrm{p}$ (away): Proportion of trials in which participants moved away as opposed to toward the emotional individual. (a) Proportion in each posture and session (session 1: baseline, session 2: with posture). Contracted postures significantly increased avoidance of anger, whereas expansive postures induced no significant change. In response to fear, there were no significant changes. (b) Change in proportion of choice from session 1 to session 2 in each posture. Only the change in response to anger in the contracted posture was significant. A confidence interval not overlapping with 0 indicates a significant change. ${ }^{*}=p<0.05$ in within-subject (colour) or between-subject (black) ttests.

Figure 3 further illustrates that this change was more strongly driven by responses to anger, than responses to fear. In participants who adopted contracted postures, the probability of choosing the chair away from the angry individual increased by $2.71 \%$ on average, but only decreased by $1.23 \%$ for fear. In participants who adopted expansive postures, there were no substantial changes for either anger $(0.28 \%)$ or fear $(-0.23 \%$, see Table 1 for means and confidence intervals).

To assess the significance of these changes from session 1 to 2 in each posture group, we compared the emotion by session interaction when the expansive and contracted group was set as the baseline category of the treatment contrast, respectively. This interaction was only significant in the contracted group $(\mathrm{OR}=1.14,95 \% \mathrm{Cl}=1.02-1.27$, $z=2.30, p=0.022$, expansive group: $O R=0.96,95 \% \mathrm{Cl}=0.86-1.07, z=-0.78, p=0.435$ ) suggesting that only contracted postures induced a significant change. Indeed, the main effect of emotion was not significant in the contracted group in session 1, but highly significant when participants adopted the contracted posture during session 2 . In the expansive group, the effect of emotion was significant in session 1, but not anymore in session 2 (see Supplementary Table S1 for details). Similar odds ratios for the emotion effect in both groups in session $1(\mathrm{OR}=1.07$ and $\mathrm{OR}=1.09)$ indicate that the emotion effect was comparably large at baseline in both groups. 


\begin{tabular}{lllllll}
\hline Session & Group & Emotion & $\mathbf{n}$ & Mean & SD & 95\% Cl \\
\hline 1- Baseline & Expansive & Anger & 40 & 52.62 & 4.1 & $51.44-53.79$ \\
1- Baseline & Expansive & Fear & 40 & 50.44 & 3.86 & $49.26-51.63$ \\
1- Baseline & Contracted & Anger & 39 & 51.86 & 5.13 & $50.51-53.21$ \\
1- Baseline & Contracted & Fear & 39 & 51.07 & 4.12 & $49.69-52.46$ \\
2- Posture & Expansive & Anger & 40 & 52.34 & 5.54 & $51.03-53.65$ \\
2 - Posture & Expansive & Fear & 40 & 50.67 & 4.33 & $49.01-52.33$ \\
2- Posture & Contracted & Anger & 39 & 54.57 & 6.47 & $53.03-56.11$ \\
2- Posture & Contracted & Fear & 39 & 49.77 & 3.56 & $48.59-50.96$ \\
\hline
\end{tabular}

Table 1. Descriptive statistics and confidence intervals for proportion of away choices. Percent of trials in which participants chose the chair away from the angry or fearful individual. Within-subject $95 \%$ confidence intervals were calculated separately for each group according to the Cousineau-Morey method.

Comparing the emotion by posture interaction with session 1 and 2 as baseline of the contrast, respectively, confirmed that the effect of emotion did not differ between groups in session $1(\mathrm{OR}=0.99,95 \% \mathrm{Cl} 0.88-1.10, \mathrm{z}=-0.27, \mathrm{p}=0.789)$, but in session 2 (OR= $1.17,95 \% \mathrm{Cl}=1.05-1.30, \mathrm{z}=2.81, \mathrm{p}=0.005)$. This suggests that the difference in session 2 was not due to baseline group differences in proportion of away vs. toward choices. Finally, neither the simple effects of session and posture, nor the session by posture interaction were significant regardless of which posture or session was set as baseline for the respective contrasts. This indicates that there were no general differences in proportion of choice between sessions or groups (e.g. more avoidance than approach in general; see Table S1 for all model estimates and significance tests).

In summary, approach and avoidance decisions in response to angry and fearful individuals did not differ between the two groups before participants adopted a posture. All participants avoided angry more than fearful individuals. After adopting a contracted posture, the probability to avoid anger as compared to fear became larger, whereas expansive postures induced no significant changes in action choices.

\section{Sensitivity analysis}

Investigating effects of emotion, session and posture on choice with an ANOVA (see SI) yielded the same results as the generalized linear mixed-effects model. The effect-size of the emotion $x$ session $x$ posture interaction in the ANOVA on choice $(F(1,77)=7.05$, $\left.\mathrm{p}=0.010, \eta^{2}{ }_{p}=0.084, \eta^{2}{ }_{G}=0.014\right)$ was slightly smaller than the minimal effect-size of $\eta^{2}{ }_{p}=0.10$ which we could detect with $80 \%$ power with our sample size. Assuming that the detected effect size corresponds to the true effect-size, this implies that we had a chance of approximately $75 \%$ to detect different behavioural changes in the two posture groups in response to the two emotions (such as the difference in avoidance of anger and fear getting larger in one posture group, but smaller in the other).

\section{Initiation time and movement duration}


The LMEMs for movement kinematics revealed no significant difference between angry and fearful trials, and no interaction of emotion with posture (see SI). Grouping anger and fear together to compare threat with neutral trials revealed quicker initiation time and slower movement duration in response to threat (see Figure 4). This suggests that threat-related facial expressions significantly reduced the time needed to initiate an action. More details and other results for movement kinematics are reported in the SI.

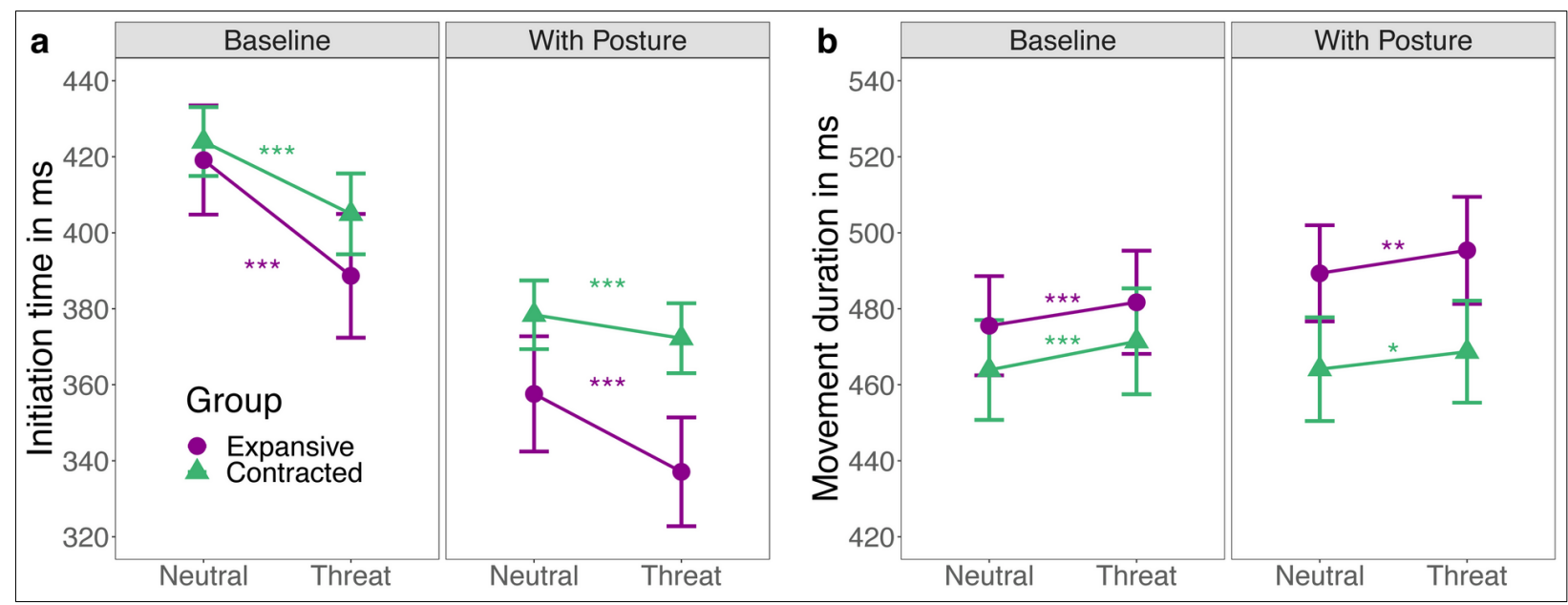

Fig 4. Initiation time and movement duration.

Means and within-subject confidence intervals for (a) initiation time (from presentation of scene to click) and (b) movement duration (from click until release on top of a chair) in ms per posture and session (session 1: baseline without posture, session 2: with posture). (a) In both sessions and groups, initiation time was quicker for trials in which one actor expressed threat (fear or anger), as compared to trials with two neutral actors. Initiation times were further quicker in the second session, with a stronger acceleration in the expansive group. (b) In both sessions and groups, movement duration was significantly longer for trials in which one actor expressed threat (fear or anger), as compared to trials with two neutral actors. In the expansive group, movement duration was further slower in the second compared to the baseline session. For neither (a) nor (b), any between-posture comparisons were significant. Note that asterisks indicate significance of the threat effect in model comparisons at * $p<0.05 ;{ }^{* *} p<.01$ and ${ }^{*} \mathrm{p}<0.001$. See Table S7 for all other effects.

\section{Discussion}

The current study assessed whether adopting expansive or contracted postures, which function as social signals of power, impacts individuals' approach and avoidance decisions in response to threat signals emitted by other individuals. More specifically, we investigated free action choices in the presence of individuals who displayed taskirrelevant angry or fearful facial expressions. Without having been informed about the emotional expressions, participants chose where to sit in a realistic scene by moving the mouse cursor to the chair of their choice, thereby either approaching or avoiding the 
emotional individual. Following a first baseline session without postures, participants repeatedly adopted either an expansive or contracted posture in between task blocks during a second session. In contrast to the outcome measures used in most previous studies assessing postural feedback effects, action decisions in response to threat signals constitute actual rather than reported behaviours related to the social signalling function of postural expansiveness. In addition, such elementary behaviours in response to task-irrelevant social stimuli are less susceptible to experimental demand effects than explicit self-reports and require no high-level cognitive processes.

The presence of angry individuals, who signal a direct threat towards the observer (Bates et al., 2015), favoured the selection of avoidance actions. This replicates previous findings from experiments that used the same task (Sander et al., 2007; Sell et al., 2014). In addition, our results suggest that power-related body postures modulated action decisions in response to task-irrelevant angry displays in the predicted directions. Specifically, contracted postures enhanced individuals' choices to avoid angry individuals $\left(d_{z}=0.38\right.$, see $\left.S I\right)$, while expansive postures decreased avoidance, but not significantly so $\left(d_{z}=-0.13\right.$, between-posture effect of $\left.d=0.51\right)$. In line with this result, the threat signal of sustained direct gaze elicits spontaneous avoidance tendencies in individuals with low power (Mennella et al., 2020; Vilarem et al., 2019). Hence, the present results could indicate that contracted postures enhance the selection of actions that avoid aggressive conspecifics.

The fact that expansive postures did not significantly decrease avoidance of angry individuals, in contrast to our predictions, is consistent with a recent meta-analysis of the power posing literature, which indicates that only contracted postures significantly change behaviour in comparison to a neutral posture (Weick et al., 2017). Due to the small number of studies including neutral conditions, we can only speculate if this applies in general, or is due to study-specific reasons, such as the context in which postures are adopted. We assessed approach-avoidance actions in the context of a waiting room. According to Guinote's (Elkjær et al., 2020) updated account of the approach motivation theory of power (2003), power activates people to pursue their goals and desires, and thus activates the approach system specifically in association with goal seeking. Therefore, expansive postures may not have increased approach behaviour because there is no goal to achieve by approaching an angry individual in the context of a waiting room. When the choice of avoiding an angry person is available and comes without any disadvantage, confronting this person may simply not be adaptive.

In the presence of fearful individuals, who simultaneously signal the presence of a potential danger and a need for affiliation (Marsh \& Ambady, 2007), participants' choices suggested neither an approach nor an avoidance preference. The changes induced by adopting expansive compared to contracted postures on approach and avoidance of fearful individuals were small and non-significant. Nonetheless, they occurred in opposite directions in the two posture groups: avoidance decreased in the contracted $\left(d_{z}=-0.15\right)$ and increased in the expansive posture group $\left(d_{z}=0.11\right)$ resulting in a small between-posture effect $(d=-0.26)$. The direction of this change, in case it reflected a true reproducible effect, would hint that participants were more sensitive to 
the affiliative than the threat aspect of fearful displays after adopting contractive compared to expansive postures, in line with research suggesting an increase in affiliation motivation in states of low power (Case et al., 2015, Guinote et al., 2015). Although we had reasonably high statistical power to detect the observed difference between angry and fearful displays (75\% for the interaction of posture and emotion), larger samples would be needed to test whether this small change in response to fearful displays (main effect of posture) are reliable.

As expected, the observed influence of postures on approach and avoidance decisions was not mediated by differences in the processing of threat-related displays. Both posture groups were quicker to initiate their actions and took longer to reach the chairs in response to both threat-related displays as compared to neutral ones, consistent with previous studies (Mennella et al., 2020; Vilarem et al., 2019). These general threat effects suggest that anger and fear displays were processed to a similar extent. Therefore, the stronger tendency to avoid angry as compared to fearful displays, which was reinforced after a contracted posture, cannot be explained by improved or quicker perceptual processing of anger as compared to fearful displays. Instead, anger and fearful displays may differentially shape action selection according to their social function (Hess et al., 2000; Springer et al., 2007; Vilarem et al., 2019). Hence, the presence of task-irrelevant angry displays more strongly favoured avoidance decisions than fearful displays because anger communicates an unambiguous aggressive intent and is perceived as a direct threat to the observer (Sell et al., 2014). Likewise, angry displays enhanced avoidance decisions after adopting a contracted as compared to an expansive posture because the communicated threat might be perceived as more challenging when the observer is in a powerless state.

Taken together, the observed effect may illustrate that our body posture constrains our action possibilities: a contracted posture may make it more difficult to confront a threatening other, and therefore lead to increased avoidance. However, increased avoidance of angry individuals could equally follow from a change in mood or effort while holding the contracted posture. Future studies could try to differentiate between these different possible mechanisms.

By demonstrating posture effects on elementary social behaviour with reasonable statistical power, the present study makes a valuable contribution to the literature. Most importantly, it is the first study investigating elementary social behaviour conceptually linked to the postures' social signalling function with relatively high statistical power. Despite the postures primary social signalling function apparent in many animal species, most previous studies focused on non-social behaviour or high-level cognitive processes, such as risky gambling, abstract thinking or sales negotiations (Cesario \& Johnson, 2017; Huang et al., 2011). The results of the present study suggest that investigating elementary social behaviour that is conceptually linked to the postures' social signalling function could be a promising direction for future research.

Second, the majority of studies on other postural feedback effects used explicit selfreports (Kozak et al., 2014; Nielsen, 2017; Peña \& Chen, 2017; Rotella \& Richeson, 2013; Strelan et al., 2014). The posture effect on explicit feelings of power in a meta- 
analysis of six pre-registered and highly-powered studies (Gronau et al., 2017) was larger in subjects familiar with "power posing", suggesting that explicit self-reports are susceptible to demand effects. Investigating the influence of postures on action decisions in response to task-irrelevant stimuli such as in the present study reduces such demand effects.

Additionally, the present study attempted to overcome some of the methodological limitations of previous studies on postural feedback effects. Past experiments that yielded significant effects tested small samples, whereas studies with large samples mostly reported null-effects (Cesario \& Johnson, 2017; Jonas et al., 2017). Moreover, very few power posing studies have so far used within-subject designs, and observed either a change only after expansive postures (Bohns \& Wiltermuth, 2012; Lee \& Schnall, 2014) or no significant effect (Jamnik \& Zvelc, 2017). Similarly, only a few studies included a control group, and observed a significant difference only for the contracted posture (Cesario \& McDonald, 2013) or no significant effects (Davis et al., 2017; Nielsen, 2017; Smith \& Apicella, 2017). Thanks to the reasonably high statistical power and to the within-subject design of the current study, we can be more confident that the observed posture effects did not originate from baseline between-group differences in approach and avoidance tendencies.

Although most previous studies do not suggest a systematic difference between male and female participants, a re-analysis of two important power posing studies (Credé \& Phillips, 2017), as well as an exploratory analysis in a highly powered study (Bombari et al., 2017), suggested that the poses' effect on feelings may be stronger in men than in women. Still, future experiments should assess whether our findings on social action decisions generalize to women.

\section{Conclusions}

This study provides first evidence that briefly adopting power-related postures could impact individuals' approach and avoidance decisions in response to social threat signals. The observed influence on action decisions in response to anger was not mediated by differences in anger processing, but reflected the social function of this emotion. Hence, postural feedback, by modulating the relevance of certain threat signals to the perceiver, may not only affect visual attention (Chadwick et al., 2019), but also the decisions taken in response to such threat signals. Within the context of the controversy around the replicability of previously published posture effects (Jonas et al., 2017; Smith \& Apicella, 2017), the present results need to be replicated before any strong conclusions can be drawn. As elementary behaviours that are conceptually linked to the social meaning of expansive and contracted postures, approach and avoidance decisions in response to threat signals may be among the most promising candidates for future replication efforts.

\section{Acknowledgements}


POSTURE EFFECTS ON SOCIAL ACTION DECISIONS

We thank Rocco Menella and Julia Maria Carbajal for advice on analysing and plotting data with linear mixed effect models. 


\section{References}

Anderson, C., \& Berdahl, J. L. (2002). The experience of power: Examining the effects of power on approach and inhibition tendencies. Journal of Personality and Social Psychology, 83(6), 1362-1377. https://doi.org/10.1037//0022-3514.83.6.1362

Andolfi, V. R., Di Nuzzo, C., \& Antonietti, A. (2017). Opening the mind through the body: The effects of posture on creative processes. Thinking Skills and Creativity, 24, 20-28. https://doi.org/10.1016/j.tsc.2017.02.012

Baguley, T. (2012). Calculating and graphing within-subject confidence intervals for ANOVA. Behavior Research Methods, 44(1), 158-175. https://doi.org/10.3758/s13428-011-0123-7

Bates, D., Mächler, M., Bolker, B., \& Walker, S. (2015). Fitting linear mixed-effects models using Ime4. Journal of Statistical Software, 67(1). https://doi.org/10.18637/jss.v067.i01

Bohns, V. K., \& Wiltermuth, S. S. (2012). It hurts when I do this (or you do that): Posture and pain tolerance. Journal of Experimental Social Psychology, 48(1), 341-345. https://doi.org/10.1016/j.jesp.2011.05.022

Bombari, D., Mast, M. S., \& Pulfrey, C. (2017). Real and imagined power poses: Is the physical experience necessary after all? Comprehensive Results in Social Psychology, 2(1), 44-54. https://doi.org/10.1080/23743603.2017.1341183

Brainard, D. H. (1997). The psychophysics toolbox. Spatial Vision, 10, 433-436.

Carney, D. R., Cuddy, A. J. C., \& Yap, A. J. (2010). Power posing: Brief nonverbal displays affect neuroendocrine levels and risk tolerance. Psychological Science, 21(10), 1363-1368. https://doi.org/10.1177/0956797610383437

Case, C. R., Conlon, K. E., \& Maner, J. K. (2015). Affiliation-seeking among the powerless: Lacking power increases social affiliative motivation. European Journal of Social Psychology, 45(3), 378-385. https://doi.org/10.1002/ejsp.2089

Cesario, J., \& Johnson, D. J. (2017). Power poseur: Bodily expansiveness does not matter in dyadic interactions. Social Psychological and Personality Science, 9(7), 781-789. https://doi.org/10.1177/1948550617725153

Cesario, J., \& McDonald, M. M. (2013). Bodies in context: Power poses as a computation of action possibility. Social Cognition, 31(2), 260-274.

Chadwick, M., Metzler, H., Tijus, C., Armony, J. L., \& Grèzes, J. (2019). Stimulus and observer characteristics jointly determine the relevance of threatening facial expressions and their interaction with attention. Motivation and Emotion, 43(2), 299-312. https://doi.org/10.1007/s11031-018-9730-2

Credé, M., \& Phillips, L. A. (2017). Revisiting the power pose effect: How robust are the results reported by Carney, Cuddy, and Yap (2010) to data analytic decisions? Social Psychological and Personality Science, 1948550617714584. https://doi.org/10.1177/1948550617714584

Davis, M. L., Papini, S., Rosenfield, D., Roelofs, K., Kolb, S., Powers, M. B., \& Smits, J. A. J. (2017). A randomized controlled study of power posing before public speaking exposure for social anxiety disorder: No evidence for augmentative 
effects. Journal of Anxiety Disorders, 52(Supplement C), 1-7. https://doi.org/10.1016/j.janxdis.2017.09.004

de Waal, F. B. M. (2007). Chimpanzee politics: Power and sex among apes (25th ed.). Johns Hopkins University Press.

Duclos, S., Laird, J., Schneider, E., Sexter, M., Stern, L., \& Vanlighten, O. (1989). Emotion-specific effects of facial expressions and postures on emotional experience. Journal of Personality and Social Psychology, 57(1), 100-108. https://doi.org/10.1037/0022-3514.57.1.100

El Zein, M., Wyart, V., \& Grèzes, J. (2015). Anxiety dissociates the adaptive functions of sensory and motor response enhancements to social threats. Elife, 4, e10274. https://doi.org/10.7554/eLife.10274

Elkjær, E., Mikkelsen, M. B., Michalak, J., Mennin, D. S., \& O'Toole, M. S. (2020). Expansive and Contractive Postures and Movement: A Systematic Review and Meta-Analysis of the Effect of Motor Displays on Affective and Behavioral Responses: Perspectives on Psychological Science. https://doi.org/10.1177/1745691620919358

Faul, F., Erdfelder, E., Lang, A.-G., \& Buchner, A. (2007). G*Power 3: A flexible statistical power analysis program for the social, behavioral, and biomedical sciences. Behavior Research Methods, 39(2), 175-191. https://doi.org/10.3758/BF03193146

Frey, S. H., \& Grafton, S. T. (2014). Finding the actor in reciprocal affordance. In The Cognitive Neurosciences (5th ed., pp. 513-520). MIT Press. https://mitpress.mit.edu/books/cognitive-neurosciences-fifth-edition

Grant, E. C., \& Mackintosh, J. H. (1963). A comparison of the social postures of some common laboratory rodents. Behaviour, 21(3), 246-259. https://doi.org/10.1163/156853963X00185

Gronau, Q. F., Erp, S. V., Heck, D. W., Cesario, J., Jonas, K. J., \& Wagenmakers, E.-J. (2017). A Bayesian model-averaged meta-analysis of the power pose effect with informed and default priors: The case of felt power. Comprehensive Results in Social Psychology, 2(1), 123-138. https://doi.org/10.1080/23743603.2017.1326760

Guinote, A. (2017). How power affects people: Activating, wanting, and goal seeking. Annual Review of Psychology, 68, 353-381.

Guinote, A., Cotzia, I., Sandhu, S., \& Siwa, P. (2015). Social status modulates prosocial behavior and egalitarianism in preschool children and adults. Proceedings of the National Academy of Sciences of the United States of America, 112(3), 731-736. https://doi.org/10.1073/pnas.1414550112

Hagelin, J. C. (2002). The kinds of traits involved in male-male competition: A comparison of plumage, behavior, and body size in quail. Behavioral Ecology, 13(1), 32-41. https://doi.org/10.1093/beheco/13.1.32

Hall, Judith. A., Coats, E. J., \& LeBeau, L. S. (2005). Nonverbal behavior and the vertical dimension of social relations: A meta-analysis. Psychological Bulletin, 131(6), 898-924. https://doi.org/10.1037/0033-2909.131.6.898 
Hess, U., Blairy, S., \& Kleck, R. E. (2000). The influence of facial emotion displays, gender, and ethnicity on judgments of dominance and affiliation. Journal of Nonverbal Behavior, 24(4), 265-283. https://doi.org/10.1023/A:1006623213355

Huang, L., Galinsky, A. D., Gruenfeld, D. H., \& Guillory, L. E. (2011). Powerful postures versus powerful roles: Which is the proximate correlate of thought and behavior? Psychological Science, 22(1), 95-102. https://doi.org/10.1177/0956797610391912

Hwang, H. C., \& Matsumoto, D. (2014). Dominance threat display for victory and achievement in competition context. Motivation and Emotion, 38(2), 206-214. https://doi.org/10.1007/s11031-013-9390-1

Jamnik, J., \& Zvelc, G. (2017). The embodiment of power and visual dominance behaviour. Interdisciplinary Description of Complex Systems, 15(4), 228-241. https://doi.org/10.7906/indecs.15.4.1

Jonas, K. J., Cesario, J., Alger, M., Bailey, A. H., Bombari, D., Carney, D., Dovidio, J. F., Duffy, S., Harder, J. A., Huistee, D. van, Jackson, B., Johnson, D. J., Keller, V. N., Klaschinski, L., LaBelle, O., LaFrance, M., Latu, I. M., Morssinkhoff, M., Nault, K., ... Tybur, J. M. (2017). Power poses - where do we stand? Comprehensive Results in Social Psychology, 2(1), 139-141. https://doi.org/10.1080/23743603.2017.1342447

Keltner, D., Gruenfeld, D. H., \& Anderson, C. (2003). Power, approach, and inhibition. Psychological Review, 110(2), 265-284. https://doi.org/10.1037/0033295X.110.2.265

Kleiner, M., Brainard, D. H., Pelli, D., Ingling, A., Murray, R., \& Broussard, C. (2007). What's new in Psychtoolbox-3? Perception, 36(14), ECVP Abstract Supplement.

Knutson, B. (1996). Facial expressions of emotion influence interpersonal trait inferences. Journal of Nonverbal Behavior, 20(3), 165-182. https://doi.org/10.1007/BF02281954

Kozak, M. N., Roberts, T.-A., \& Patterson, K. E. (2014). She stoops to conquer? How posture interacts with self-objectification and status to impact women's affect and performance. Psychology of Women Quarterly, 38(3), 414-424. https://doi.org/10.1177/0361684313517865

Langner, O., Dotsch, R., Bijlstra, G., Wigboldus, D. H. J., Hawk, S. T., \& van Knippenberg, A. (2010). Presentation and validation of the Radboud Faces Database. Cognition \& Emotion, 24(8), 1377-1388. https://doi.org/10.1080/02699930903485076

Lawrence, M. A. (2016). Ez: Easy analysis and visualization of factorial experiments. $R$ Package Version 4.4-0. https://CRAN.R-project.org/package=ez

Lee, E. H., \& Schnall, S. (2014). The influence of social power on weight perception. Journal of Experimental Psychology-General, 143(4), 1719-1725. https://doi.org/10.1037/a0035699

Marsh, A. A., Adams, R. B., \& Kleck, R. E. (2005). Why do fear and anger look the way they do? Form and social function in facial expressions. Personality and Social Psychology Bulletin, 31(1), 73-86. https://doi.org/10.1177/0146167204271306 
Marsh, A. A., \& Ambady, N. (2007). The influence of the fear facial expression on prosocial responding. Cognition \& Emotion, 21(2), 225-247. https://doi.org/10.1080/02699930600652234

MathWorks, Inc. (2014). Matlab and Statistics Toolbox release 2014b.

Mehrabian, A. (1981). Silent messages. Wadsworth.

Mennella, R., Vilarem, E., \& Grèzes, J. (2020). Rapid approach-avoidance responses to emotional displays reflect value-based decisions: Neural evidence from an EEG study. Neuroimage.

Metzler, H. (2018). Does your body affect what you see? No power posture effects on explicit recognition of threat-related facial expressions. In Doctoral dissertation: The influence of bodily actions on social perception and behaviour: Assessing effects of power postures (pp. 67-82). Sorbonne Université.

Niedenthal, P. M. (2007). Embodying emotion. Science, 316(5827), 1002-1005. https://doi.org/10.1126/science.1136930

Nielsen, S. K. (2017). Posture and social problem solving, self-esteem, and optimism. International Journal of Psychological Studies, 9(4), 44. https://doi.org/10.5539/ijps.v9n4p44

Paulus, A., \& Wentura, D. (2016). It depends: Approach and avoidance reactions to emotional expressions are influenced by the contrast emotions presented in the task. Journal of Experimental Psychology. Human Perception and Performance, 42(2), 197-212. https://doi.org/10.1037/xhp0000130

Peña, J., \& Chen, M. (2017). With great power comes great responsibility: Superhero primes and expansive poses influence prosocial behavior after a motion-controlled game task. Computers in Human Behavior, 76, 378-385. https://doi.org/10.1016/j.chb.2017.07.039

R Core Team. (2018). R: A language and environment for statistical computing. R Foundation for Statistical Computing. https://www.R-project.org/

Revelle, W. (2017). psych: Procedures for psychological, psychometric, and personality research. $R$ Package Version 1.7.5. https://CRAN.R-project.org/package=psych

Riskind, J. H. (1984). They stoop to conquer-Guiding and self-regulatory functions of physical posture after success and failure. Journal of Personality and Social Psychology, 47(3), 479-493. https://doi.org/10.1037/0022-3514.47.3.479

Riskind, J. H., \& Gotay, C. C. (1982). Physical posture: Could it have regulatory or feedback effects on motivation and emotion? Motivation and Emotion, 6(3), 273298.

Rotella, K. N., \& Richeson, J. A. (2013). Body of guilt: Using embodied cognition to mitigate backlash to reminders of personal \& ingroup wrongdoing. Journal of Experimental Social Psychology, 49(4), 643-650. https://doi.org/10.1016/j.jesp.2013.02.013

Sander, D., Grandjean, D., Kaiser, S., Wehrle, T., \& Scherer, K. R. (2007). Interaction effects of perceived gaze direction and dynamic facial expression: Evidence for appraisal theories of emotion. European Journal of Cognitive Psychology, 19(3), 470-480. https://doi.org/10.1080/09541440600757426 
Schenkel, R. (1967). Submission-Its features and function in wolf and dog. American Zoologist, 7(2), 319-329.

Schuett, G. W. (1997). Body size and agonistic experience affect dominance and mating success in male copperheads. Animal Behaviour, 54(1), 213-224. https://doi.org/10.1006/anbe.1996.0417

Schultheiss, O. C., \& Hale, J. A. (2007). Implicit motives modulate attentional orienting to facial expressions of emotion. Motivation and Emotion, 31(1), 13-24. https://doi.org/10.1007/s11031-006-9042-9

Sell, A., Cosmides, L., \& Tooby, J. (2014). The human anger face evolved to enhance cues of strength. Evolution and Human Behavior, 35(5), 425-429. https://doi.org/10.1016/j.evolhumbehav.2014.05.008

Sell, A., Cosmides, L., Tooby, J., Sznycer, D., von Rueden, C., \& Gurven, M. (2009). Human adaptations for the visual assessment of strength and fighting ability from the body and face. Proceedings of the Royal Society B-Biological Sciences, 276(1656), 575-584. https://doi.org/10.1098/rspb.2008.1177

Simonsohn, U. (2015). [17] No-way interactions. The Winnower, 5, e142559.90552. https://doi.org/10.15200/winn.142559.90552

Smith, K. M., \& Apicella, C. L. (2017). Winners, losers, and posers: The effect of power poses on testosterone and risk-taking following competition. Hormones and Behavior, 92. https://doi.org/10.1016/j.yhbeh.2016.11.003

Springer, U. S., Rosas, A., McGetrick, J., \& Bowers, D. (2007). Differences in startle reactivity during the perception of angry and fearful faces. Emotion, 7(3), 516-525. https://doi.org/10.1037/1528-3542.7.3.516

Stepper, S., \& Strack, F. (1993). Proprioceptive determinants of emotional and nonemotional feelings. Journal of Personality and Social Psychology, 64(2), 211.

Stevenson, P. A., Hofmann, H. A., Schoch, K., \& Schildberger, K. (2000). The fight and flight responses of crickets depleted of biogenic amines. Journal of Neurobiology, 43(2), 107-120.

Strelan, P., Weick, M., \& Vasiljevic, M. (2014). Power and revenge. British Journal of Social Psychology, 53(3), 521-540. https://doi.org/10.1111/bjso.12044

Tokarz, R. R. (1985). Body size as a factor determining dominance in staged agonistic encounters between male brown anoles (Anolis sagrei). Animal Behaviour, 33(3), 746-753. https://doi.org/10.1016/S0003-3472(85)80006-3

Tracy, J. L., \& Matsumoto, D. (2008). The spontaneous expression of pride and shame: Evidence for biologically innate nonverbal displays. Proceedings of the National Academy of Sciences of the United States of America, 105(33), 11655-11660. https://doi.org/10.1073/pnas.0802686105

Vetter, P., Badde, S., Phelps, E. A., \& Carrasco, M. (2019). Emotional faces guide the eyes in the absence of awareness. ELife, 8, e43467. https://doi.org/10.7554/eLife.43467

Vilarem, E., Armony, J. L., \& Grèzes, J. (2019). Action opportunities modulate attention allocation under social threat. Emotion, Advance online publication. https://doi.org/10.1037/emo0000598 
Weick, M., McCall, C., \& Blascovich, J. (2017). Power moves beyond complementarity: A staring look elicits avoidance in low power perceivers and approach in high power perceivers. Personality and Social Psychology Bulletin, 43(8), 1188-1201. https://doi.org/10.1177/0146167217708576

Weisfeld, G. E., \& Beresford, J. M. (1982). Erectness of posture as an indicator of dominance or success in humans. Motivation and Emotion, 6(2), 113-131. https://doi.org/10.1007/BF00992459

Wickham, H. (2009). ggplot2: Elegant graphics for data analysis. Springer-Verlag New York. http://ggplot2.org

Wickham, H., Francois, R., Henry, L., \& Müller, K. (2017). dplyr: A grammar of data manipulation. $R$ Package Version 0.7.4. https://CRAN.R-project.org/package $=d$ plyr

Wickham, H., \& Henry, L. (2018). tidyr: Easily tidy data with "spread()" and "gather()" functions. $R$ Package Version 0.8.1. https://CRAN.R-project.org/package=tidyr

Willis, G. B., Rodríguez-Bailón, R., \& Lupiáñez, J. (2011). The boss is paying attention: Power affects the functioning of the attentional networks. Social Cognition, 29(2), 166-181. https://doi.org/10.1521/soco.2011.29.2.166

Yap, A. J., Wazlawek, A. S., Lucas, B. J., Cuddy, A. J. C., \& Carney, D. R. (2013). The ergonomics of dishonesty: The effect of incidental posture on stealing, cheating, and traffic violations. Psychological Science, 24(11), 2281-2289. https://doi.org/10.1177/0956797613492425 